FACTA UNIVERSITATIS (NIŠ)

Ser. Math. Inform. Vol. 36, No 2 (2021), 419-434

https://doi.org/10.22190/FUMI200923031P

Original Scientific Paper

\title{
$\eta$-RICCI SOLITONS ON LORENTZIAN PARA-KENMOTSU MANIFOLDS
}

\author{
Shashikant Pandey ${ }^{1}$, Abhishek Singh ${ }^{1}$ and Vishnu Narayan Mishra ${ }^{2}$ \\ 1 Faculty of Science, Department of Mathematics and Astronomy, \\ University of Lucknow, 226007 Lcknow, Uttar Pradesh, India \\ 2 Faculty of Science, Department of Mathematics, \\ Indira Gandhi National Tribal University, Lalpur, Amarkantak \\ 484887, Madhya Pradesh, Amarkantak, India
}

\begin{abstract}
The objective of present research article is to investigate the geometric properties of $\eta$-Ricci solitons on Lorentzian para-Kenmotsu manifolds. In this manner, we consider $\eta$-Ricci solitons on Lorentzian para-Kenmotsu manifolds satisfying $R \cdot S=0$. Further, we obtain results for $\eta$-Ricci solitons on Lorentzian para-Kenmotsu manifolds with quasi-conformally flat property. Moreover, we get results for $\eta$-Ricci solitons in Lorentzian para-Kenmotsu manifolds admitting Codazzi type of Ricci tensor and cyclic parallel Ricci tensor, $\eta$-quasi-conformally semi-symmetric, $\eta$-Ricci symmetric and quasiconformally Ricci semi-symmetric. At last, we construct an example of a such manifold which justify the existence of proper $\eta$-Ricci solitons.

Key words: $\eta$-Ricci solitons; Lorentzian Para-Kenmotsu manifolds; Codazzi type of Ricci tensor; Cyclic parallel Ricci tensor; quasi-conformal curvature tensor.
\end{abstract}

\section{Introduction}

In 1982, Hamilton [17] introduced the notion of the Ricci flow to find a canonical metric on a smooth manifold. The Ricci flow is an evolution equation for metrics

Received September 23, 2020; accepted January 26, 2021

Communicated by Uday Chand De

Corresponding Author: Vishnu Narayan Mishra, Faculty of Science, Department of Mathematics, Indira Gandhi National Tribal University, Lalpur, Amarkantak, 484887, Madhya Pradesh, Amarkantak, India | E-mail: vishnunarayanmishra@gmail.com 2010 Mathematics Subject Classification. 53C15, 53C25.

(C) 2021 by University of Niš, Serbia | Creative Commons License: CC BY-NC-ND 
on a Riemannian manifold

$$
\frac{\partial}{\partial t} g_{i j}(t)=-2 R_{i j}
$$

A Ricci soliton is a natural generalization of an Einstein metric and is defined on a Riemannian manifold $(M, g)[6]$. A Ricci soliton is a triple $(g, V, \lambda)$ with $g$ a Riemannian metric, $V$ a vector field (called the potential vector field), and $\lambda$ a real scalar such that

$$
£_{V} g+2 S+2 \lambda g=0,
$$

where $S$ is a Ricci tensor of $M$ and $£_{V}$ denotes the Lie derivative operator along the vector field $V$. The Ricci soliton is said to be shrinking, steady and expanding accordingly as $\lambda$ is negative, zero and positive, respectively [18]. A Ricci soliton with $V$ zero is reduced to Einstein equation. Metrics satisfying (1.2) is interesting and useful in physics and is often referred as quasi-Einstein [22, 23]. Compact Ricci solitons are the fixed points of the Ricci flow $\frac{\partial}{\partial t} g=-2 S$, projected from the space of metrics onto its quotient modulo diffeomorphisms and scalings, and often arise blow-up limits for the Ricci flow on compact manifolds. Theoretical physicists have also been looking into the equation of Ricci soliton in relation with string theory. The initial contributions in this direction is due to Friedmann [7], who discusses some aspects of it. Ricci solitons were introduced in Riemannian geometry [17], as the self-similar solutions of the Ricci flow, and play an important role in understanding its singularities. Ricci solitons have been studied in many contexts by several authors such as $[24,25,26,19,21]$ and many others.

As a generalization of Ricci soliton, the notion of $\eta$-Ricci soliton introduced by J. T. Cho and M. Kimura [9], which was treated by C. Calin and M. Crasmareanu on Hopf hypersurfaces in complex space forms [6]. An $\eta$-Ricci soliton is a tuple $(g, V, \lambda, \mu)$, where $V$ is a vector field on $M$, and $\lambda$ are $\mu$ constants and $g$ is a Riemannian (or pseudo-Riemannian) metric satisfying the equation

$$
£_{V} g+2 S+2 \lambda g+2 \mu \eta \otimes \eta=0,
$$

where $S$ is the Ricci tensor associated to $g$.

In particular, if $\mu=0$, then the notion of $\eta$-Ricci solitons $(g, V, \lambda, \mu)$ reduces to the notion of Ricci solitons $(g, V, \lambda)$. If $\mu \neq 0$, then the $\eta$-Ricci solitons are called proper $\eta$-Ricci solitons. We refer to $[28,14,10]$ and references therein for a survey and further references on the geometry of Ricci solitons on pseudo-Riemannian manifolds.

Recently an $\eta$-Ricci soliton has been studied by several authors such as $[3,5$, $16,20,8,4]$ and they found many interesting geometric properties.

These above results motivated me to study $\eta$-Ricci solitons on Lorentzian paraKenmotsu manifolds satisfying certain curvature conditions. The paper is organized in the following way. In Section 2, we give a brief introduction of an Lorentzian para-Kenmotsu manifold. Section 3 deals with the study of Ricci solitons and $\eta$-Ricci solitons in Lorentzian para-Kenmotsu manifolds. In Section 4, we study $\eta$ Ricci solitons on Lorentzian para-Kenmotsu manifolds satisfying $R \cdot S=0 . \eta$-Ricci 
solitons on quasi-conformally flat Lorentzian para-Kenmotsu manifolds have been studied in Section 5. In Section 6, we study $\eta$-Ricci solitons in Lorentzian paraKenmotsu manifolds admitting Codazzi type of Ricci tensor and cyclic parallel Ricci tensor. Section 7 is devoted to the study of $\varphi$-quasi-conformally semi-symmetric $\eta$-Ricci solitons on Lorentzian para-Kenmotsu manifolds. Beside these we study $\eta$-Ricci solitons on $\varphi$-Ricci symmetric Lorentzian para-Kenmotsu manifolds. Also $\eta$-Ricci solitons on conformally Ricci semi-symmetric Lorentzian para-Kenmotsu manifolds has been considered. Finally, we construct a 3-dimensional example of a Lorentzian para-Kenmotsu manifold which admits an $\eta$-Ricci soliton.

\section{Preliminaries}

An $n$-dimensional differential manifold $M$ is a Lorentzian metric manifold. If it admits a $(1,1)$-tensor field $\varphi$, contravariant vector field $\xi$, a covariant vector field $\eta$ and a Lorentzian metric $g$, which satisfy [15]:

$$
\varphi^{2} X=X+\eta(X) \xi, \quad \eta(\xi)=-1,
$$

which implies

$$
\text { (a) } \varphi \xi=0, \quad(b) \quad \eta(\varphi X)=0, \quad(c) \operatorname{rank}(\varphi)=n-1 .
$$

Then $M$ admits a Lorentzian metric $g$, such that

$$
g(\varphi X, \varphi Y)=g(X, Y)+\eta(X) \eta(Y),
$$

and $M$ is said to admit a Lorentzian almost paracontact structure $(\varphi, \xi, \eta, g)$. In this case, we have

$$
\text { (a) } g(X, \xi)=\eta(X), \quad \text { (b) } \nabla_{X} \xi=\varphi X,
$$

$$
\left(\nabla_{X} \varphi\right) Y=g(X, Y) \xi+\eta(Y) X+2 \eta(X) \eta(Y) \xi
$$

where $\nabla$ denotes the operator of covariant differentiation with respect to the Lorentzian metric $g$.

If we put

$$
\Omega(X, Y)=g(X, \varphi Y)=g(\varphi X, Y)=\Omega(Y, X),
$$

for any vector fields $X$ and $Y$, then the tensor field $\Omega(X, Y)$ is a symmetric $(0,2)$ tensor field.

Recently, Haseeb and Prasad define a new manifold called Lorentzian paraKenmostu manifold [1].

Definition 2.1. A Lorentzian almost paracontact manifold $M$ is called Lorentzian para-Kenmostu manifold if

$$
\left(\nabla_{X} \varphi\right) Y=-g(\varphi X, Y) \xi-\eta(Y) \varphi X,
$$

for any vector fields $X, Y$ on $M[1]$. 
In the Lorentzian para-Kenmostu manifold, we have

$$
\begin{gathered}
\nabla_{X} \xi=-X-\eta(X) \xi \\
\left(\nabla_{X} \eta\right)(Y)=-g(X, Y)-\eta(X) \eta(Y) .
\end{gathered}
$$

Also in an Lorentzian para-Kenmostu manifold $M$, the following relations hold:

$$
\begin{gathered}
\eta\left(\nabla_{X} \xi\right)=0, \quad \nabla_{\xi} \xi=0 \\
g(R(X, Y) Z, \xi)=\eta(R(X, Y) Z)=g(Y, Z) \eta(X)-g(X, Z) \eta(Y), \\
R(X, Y) \xi=\eta(Y) X-\eta(X) Y, \\
R(\xi, X) Y=g(X, Y) \xi-\eta(Y) X, \\
R(\xi, X) \xi=X+\eta(X) \xi \\
S(X, \xi)=(n-1) \eta(X), \\
Q \xi=(n-1) \xi
\end{gathered}
$$

for any $X, Y, Z$ on $M$.

Definition 2.2. The quasi-conformal curvature tensor $W$ in a Lorentzian paraKenmotsu manifold $M$ is defined by

$$
\begin{aligned}
W(X, Y) Z= & a R(X, Y) Z+b(S(Y, Z) X-S(X, Z) Y \\
& +g(Y, Z) Q X-g(X, Z) Q Y) \\
& -\frac{r}{n}\left(\frac{a}{n-1}+2 b\right)(g(Y, Z) X-g(X, Z) Y),
\end{aligned}
$$

where $a$ and $b$ are constants such that $a b \neq 0$ and $R, S, Q$, and $r$ are the Riemannian curvature tensor of type $(1,3)$, the Ricci tensor of type $(0,2)$, the Ricci operator defined by $g(Q X, Y)=S(X, Y)$, and the scalar curvature of the manifold, respectively (see $[12])$.

$$
\text { If } a=1 \text { and } b=-\frac{1}{(n-2)} \text {, then (2.17) takes the form }
$$

$$
\begin{aligned}
W(X, Y) Z= & R(X, Y)-\frac{1}{(n-2)}(S(Y, Z) X-S(X, Z) Y \\
& +g(Y, Z) Q X-g(X, Z) Q Y) \\
& -\frac{r}{(n-1)(n-2)}(g(Y, Z) X-g(X, Z) Y) \\
= & C(X, Y) Z,
\end{aligned}
$$

where $C$ is the conformal curvature tensor [13,11]. Thus the conformal curvature tensor $C$ is a particular case of a quasi-conformal curvature tensor $W$. The manifold is said to be quasi-conformally flat if $W$ vanishes identically on $M$.

Definition 2.3. If $(M, V, \lambda, \mu)$ is an $\eta$-Ricci soliton, then the 1 -form $\xi$ is said to be a potential vector field. 


\section{Ricci and $\eta$-Ricci solitons on Lorentzian para-Kenmotsu manifolds}

Let $(M, \varphi, \xi, \eta, g)$ be an paracontact metric manifolds. Consider the equation

$$
£_{\xi} g+2 S+2 \lambda g+2 \mu \eta \otimes \eta=0
$$

where $£_{\xi}$ is the Lie derivative operator along the vector field $\xi, S$ is the Ricci curvature tensor field of the metric $g$, and $\lambda$ and $\mu$ are real constants. Writing $£_{\xi} g$ in terms of the Levi-Civita connection $\nabla$, we have:

$$
2 S(X, Y)=-g\left(\nabla_{X} \xi, Y\right)-g\left(X, \nabla_{Y} \xi\right)-2 \lambda g(X, Y)-2 \mu \eta(X) \eta(Y),
$$

for any $X, Y \in \chi(M)$, or equivalent:

$$
S(X, Y)=(1-\lambda) g(X, Y)+(1-\mu) \eta(X) \eta(Y),
$$

for any $X, Y \in \chi(M)$.

The above equation yields

$$
\begin{gathered}
S(X, \xi)=(\mu-\lambda) \eta(X), \\
Q X=(1-\lambda) X+(1-\mu) \eta(X) .
\end{gathered}
$$

Comparing (2.15) with (3.4), we get

$$
\mu-\lambda=n-1 .
$$

The data $(g, \xi, \lambda, \mu)$ which satisfy the equation (3.1) is said to be an $\eta$-Ricci soliton on $M[9]$.

Thus, we can state the following theorem:

Theorem 3.1. Let $M$ be an n-dimensional Lorentzian para-Kenmotsu manifold. If the manifold admits an $\eta$-Ricci soliton $(g, \xi, \lambda, \mu)$, then $M$ an $\eta$-Einstein manifold of the form (3.3), and the scalars $\lambda$ and $\mu$ are related by $\mu-\lambda=n-1$.

In particular, if we take $\mu=0$ in (3.3) and (3.6), then we obtain $S(X, Y)=$ $(1-\lambda) g(X, Y)+\eta(X) \eta(Y)$, and $\lambda=1-n$, respectively. Thus, we have

Corollary 3.1. Let $M$ be an n-dimensional Lorentzian para-Kenmotsu manifold. If the manifold admits a Ricci soliton $(g, \xi, \lambda)$, then $M$ is an $\eta$-Einstein manifold and the manifold is expanding or shrinking according to the vector field $\xi$ being spacelike or timelike. 


\section{4. $\eta$-Ricci solitons on Lorentzian para-Kenmotsu manifolds satisfying $R \cdot S=0$}

In this section we are going to study, an $n$-dimensional Lorentzian para-Kenmotsu manifold admitting an $\eta$-Ricci soliton satisfies $R \cdot S=0$, which implies

$$
(R(X, Y) \cdot S)(Z, W)=0 .
$$

From (4.1), we have

$$
S(R(X, Y) Z, W)+S(Z, R(X, Y) W)=0 .
$$

Putting $X=\xi$ in (4.2), we obtain

$$
S(R(\xi, Y) Z, W)+S(Z, R(\xi, Y) W)=0 .
$$

Replacing the expression of $S$ from (3.3) and from the symmetries of $R$, we find

$$
(\mu-1)[\eta(Y) g(X, Z)+\eta(Z) g(X, Y)+2 \eta(X) \eta(Y) \eta(Z)]=0,
$$

taking $Z=\xi$ in (4.4), we have

$$
(\mu-1)[g(X, Y)+\eta(X) \eta(Y)]=0,
$$

from which it follows that $\mu=1$. From the relation (3.6), we get $\lambda=(2-n)$.

Thus, we can state the following theorem:

Theorem 4.1. Let $(g, \xi, \lambda, \mu)$ be an n-dimensional Lorentzian para-Kenmotsu manifold admitting a proper $\eta$-Ricci soliton satisfies $R \cdot S=0$, then $\mu=1$ and $\lambda=(2-n)$.

From the above theorem we get:

Corollary 4.1. On a Lorentzian para-Kenmotsu manifold $M$ satisfying $R \cdot S=0$, there is no Ricci soliton with the potential vector field $\xi$.

\section{5. $\eta$-Ricci solitons on quasi-conformally flat Lorentzian para-Kenmotsu manifolds}

In this constituent we review an $\eta$-Ricci solitons on quasi-conformally flat Lorentzian para-Kenmotsu manifolds.

Let us assume that the manifold $M$ admitting $\eta$-Ricci solitons is quasi-conformally flat, that is, $W=0$. Then, from (2.17) it follows that

$$
\begin{aligned}
R(X, Y) Z= & -\frac{b}{a}(S(Y, Z) X-S(X, Z) Y \\
& +g(Y, Z) Q X-g(X, Z) Q Y) \\
& -\frac{r}{a n}\left(\frac{a}{n-1}+2 b\right)(g(Y, Z) X-g(X, Z) Y) .
\end{aligned}
$$


Taking the inner product of (5.1) with $\xi$ and using (2.4), (3.3) and (3.4), we get

$$
\begin{aligned}
\eta(R(X, Y) Z)= & {\left[\frac{\lambda b}{a}-\frac{n b}{a}+\frac{r}{a n}\left(\frac{a}{n-1}+2 b\right)\right] } \\
& \cdot[g(Y, Z) \eta(X)-g(X, Z) \eta(Y)] .
\end{aligned}
$$

By virtue of (2.11) and (5.3), we obtain

$$
\left[\frac{\lambda b}{a}-\frac{n b}{a}+\frac{r}{a n}\left(\frac{a}{n-1}+2 b\right)-1\right][g(Y, Z) \eta(X)-g(X, Z) \eta(Y)]=0 .
$$

Now, putting $X=\xi$ in the last equation, we find

$$
\left[\frac{\lambda b}{a}-\frac{n b}{a}+\frac{r}{a n}\left(\frac{a}{n-1}+2 b\right)-1\right][g(Y, Z)+\eta(Y) \eta(Z)]=0,
$$

from which it follows that $\lambda=\left[n+\frac{a}{b}-\frac{r}{b n}\left(\frac{a}{n-1}+2 b\right)\right]$.

From the relation (3.6), we obtain $\mu=\left[2 n+\frac{a}{b}-\frac{r}{b n}\left(\frac{a}{n-1}+2 b\right)-1\right]$.

Thus, we can state the following theorem:

Theorem 5.1. A quasi-conformally flat Lorentzian para-Kenmotsu manifold admits a proper $\eta$-Ricci solitons with $\lambda=\frac{a}{b}\left(1-\frac{r}{n(n-1)}\right)+\left(n-\frac{2 r}{n}\right)$ and $\mu=\frac{a}{b}\left(1-\frac{r}{n(n-1)}\right)+\left(2 n-\frac{2 r}{n}-1\right)$.

As a corollary of this theorem we have:

Corollary 5.1. On a Lorentzian para-Kenmotsu manifold satisfying $W=0$, there is no Ricci soliton with the potential vector field $\xi$.

\section{6. $\quad \eta$-Ricci solitons on Lorentzian para-Kenmotsu manifolds admitting Codazzi type of Ricci tensor and cyclic parallel Ricci tensor}

In this section we consider $\eta$-Ricci solitons in Lorentzian para-Kenmotsu manifolds admitting Codazzi type of Ricci tensor and cyclic parallel Ricci tensor. Gray [2] introduced the notion of cyclic parallel Ricci tensor and Codazzi type of Ricci tensor.

Definition 6.1. A Lorentzian para-Kenmotsu manifold is said to have Codazzi type of Ricci tensor if its Ricci tensor $S$ of type $(0,2)$ is non-zero and satisfies the following condition

$$
\left(\nabla_{Z} S\right)(X, Y)=\left(\nabla_{X} S\right)(Y, Z)
$$

for all $X, Y, Z$ on $M$. 
Taking covariant derivative of (3.3) along $Z$, we get

$$
\left(\nabla_{Z} S\right)(X, Y)=(1-\mu)\left[\left(\nabla_{Z} \eta\right)(X) \eta(Y)+\eta(X)\left(\nabla_{Z} \eta\right)(Y)\right]
$$

by virtue of (2.9) and (6.2), we obtain

(6.3) $\left(\nabla_{Z} S\right)(X, Y)=(\mu-1)[g(Z, X) \eta(Y)+g(Z, Y) \eta(X)+2 \eta(X) \eta(Y) \eta(Z)]$.

By hypothesis the Ricci tensor $S$ is of Coddazi type. Then

$$
\left(\nabla_{Z} S\right)(X, Y)=\left(\nabla_{X} S\right)(Y, Z) .
$$

Making use of (6.3), (6.4) takes the form

$$
(\mu-1)[g(Z, Y) \eta(X)-g(X, Y) \eta(Z)]=0 .
$$

Substituting $Z=\xi$ in $(6.5)$, we find

$$
(\mu-1)[\eta(Y) \eta(X)+g(X, Y)]=0,
$$

from which it follows that $\mu=1$. From the relation (3.6), we obtain $\lambda=(2-n)$.

Thus, we can state the following theorem:

Theorem 6.1. Let $(g, \xi, \lambda, \mu)$ be a proper $\eta$-Ricci soliton in an $n$-dimensional Lorentzian para-Kenmotsu manifold. If the manifold has Ricci tensor of Codazzi type, then $\mu=1$ and $\lambda=(2-n)$.

From the above theorem we have:

Corollary 6.1. A Lorentzian para-Kenmotsu manifold Ricci tensor is of Codazzi type does not admit Ricci solitons with potential vector field $\xi$.

Definition 6.2. A Lorentzian para-Kenmotsu manifold is said to have cyclic parallel Ricci tensor if its Ricci tensor $S$ of type $(0,2)$ is non-zero and satisfies the following condition [18]

$$
\left(\nabla_{X} S\right)(Y, Z)+\left(\nabla_{Y} S\right)(Z, X)+\left(\nabla_{Z} S\right)(X, Y)=0
$$

for all $X, Y, Z$ on $M$.

Let $(g, \xi, \lambda, \mu)$ be an $\eta$-Ricci soliton in an $n$-dimensional Lorentzian para-Kenmotsu manifold and the manifold has cyclic parallel Ricci tensor, then (6.7) holds. Taking covariant derivative of (3.3) and making use of (2.9), we obtain

(6.8) $\left(\nabla_{Z} S\right)(X, Y)=(\mu-1)[g(Z, X) \eta(Y)+g(Z, Y) \eta(X)+2 \eta(X) \eta(Y) \eta(Z)]$.

Similarly, we have

$(6.9)\left(\nabla_{X} S\right)(Y, Z)=(\mu-1)[g(X, Y) \eta(Z)+g(X, Z) \eta(Y)+2 \eta(X) \eta(Y) \eta(Z)]$, 
and

$(6.10)\left(\nabla_{Y} S\right)(Z, X)=(\mu-1)[g(Y, Z) \eta(X)+g(Y, X) \eta(Z)+2 \eta(X) \eta(Y) \eta(Z)]$.

By using (6.8)-(6.10) in (6.10), we find

$(6.12() \mu-1)[g(X, Y) \eta(Z)+g(Y, Z) \eta(X)+g(Z, X) \eta(Y)+3 \eta(X) \eta(Y) \eta(Z)]=0$.

Now, putting $Z=\xi$ in $(6.11)$, we have

$$
2(\mu-1)[g(X, Y)+\eta(X) \eta(Y)]=0,
$$

from which it follows that $\mu=1$. From the relation (3.6), we obtain $\lambda=(2-n)$.

Thus, we can state the following theorem:

Theorem 6.2. Let $(g, \xi, \lambda, \mu)$ be a proper $\eta$-Ricci soliton in an $n$-dimensional Lorentzian para-Kenmotsu manifold. If the manifold has cyclic parallel Ricci tensor, then $\mu=1$ and $\lambda=(2-n)$.

From the above theorem we get:

Corollary 6.2. A Lorentzian para-Kenmotsu manifold has cyclic parallel Ricci tensor does not admit Ricci solitons with potential vector field $\xi$.

\section{7. $\eta$-Ricci solitons on $\varphi$-quasi-conformally semi-symmetric Lorentzian para-Kenmotsu manifolds}

This section is devoted to the study of $\varphi$-quasi-conformally semi-symmetric $\eta$-Ricci solitons on Lorentzian para-Kenmotsu manifolds. Then, we have

$$
W \cdot \varphi=0,
$$

from which it follows that

$$
W(X, Y) \varphi Z-\varphi(W(X, Y) Z)=0 .
$$

Taking $Z=\xi$ in $(7.2)$, we have

$$
\varphi(W(X, Y) \xi)=0 .
$$

Now, replacing $Z=\xi$ in (2.17) and using (2.2), (2.12), (2.15) and (2.16), we obtain

$$
W(X, Y) \xi=\left(a+n b-\frac{r a}{n(n-1)}-\frac{2 r b}{n}-b \lambda\right)[\eta(Y) X-\eta(X) Y] .
$$

In view of (7.3) and (7.4), we get

$$
\begin{aligned}
\varphi(W(X, Y) \xi) & =\left(a+n b-\frac{r a}{n(n-1)}-\frac{2 r b}{n}-b \lambda\right)[\eta(Y) \varphi X-\eta(X) \varphi Y] \\
& =0 .
\end{aligned}
$$


Now, substituting $X$ by $\varphi X$ in (7.5), we find

$$
\left(a+n b-\frac{r a}{n(n-1)}-\frac{2 r b}{n}-b \lambda\right) \eta(Y) \varphi^{2} X=0 .
$$

By virtue of (2.1) and (7.7), we have

$$
\left(a+n b-\frac{r a}{n(n-1)}-\frac{2 r b}{n}-b \lambda\right)[X+\eta(X) \xi]=0 .
$$

Taking the inner product of (7.7) with respect to $U$, we find

$$
\left(a+n b-\frac{r a}{n(n-1)}-\frac{2 r b}{n}-b \lambda\right)[g(X, U)+\eta(X) \eta(U)]=0,
$$

from which it follows that $\lambda=\frac{a}{b}\left(1-\frac{r}{n(n-1)}\right)+\left(n-\frac{2 r}{n}\right)$. From the relation (3.6), we obtain $\mu=\frac{a}{b}\left(1-\frac{r}{n(n-1)}\right)+\left(2 n-\frac{2 r}{n}-1\right)$.

Thus, we can state the following theorem:

Theorem 7.1. A $\varphi$-conformally semi-symmetric Lorentzian para-Kenmotsu manifold admits a proper $\eta$-Ricci solitons with $\lambda=\frac{a}{b}\left(1-\frac{r}{n(n-1)}\right)+\left(n-\frac{2 r}{n}\right)$ and $\mu=\frac{a}{b}\left(1-\frac{r}{n(n-1)}\right)+\left(2 n-\frac{2 r}{n}-1\right)$.

As a corollary of this theorem we have:

Corollary 7.1. On a Lorentzian para-Kenmotsu manifold satisfying $W \cdot \varphi=0$, there is no Ricci soliton with the potential vector field $\xi$.

\section{8. $\eta$-Ricci solitons on $\varphi$-Ricci symmetric Lorentzian para-Kenmotsu manifolds}

In this section, we study $\varphi$-Ricci symmetric $\eta$-Ricci soliton on Lorentzian paraKenmotsu manifolds.

Definition 8.1. A Lorentzian para-Kenmotsu manifold is said to be $\varphi$-Ricci symmetric if

$$
\varphi^{2}\left(\nabla_{X} Q\right) Y=0
$$

holds for all smooth vector fields $X, Y$.

If $X, Y$ are orthogonal to $\xi$, then the manifold is said to be locally $\varphi$-Ricci symmetric. It is well-known that $\varphi$-symmetric implies $\varphi$-Ricci symmetric, but the converse, is not, in general true. $\varphi$-Ricci symmetric Sasakian manifolds have been studied by De and Sarkar [27]. 
We know that, the Ricci tensor for an $\eta$-Ricci soliton on Lorentzian paraKenmotsu manifold is given by

$$
S(X, Y)=(1-\lambda) g(X, Y)+(1-\mu) \eta(X) \eta(Y) .
$$

Then it follows that

$$
Q Y=(1-\lambda) Y+(1-\mu) \eta(Y) \xi
$$

for all smooth vector fields $Y$. Taking covariant derivative of above equation with respect to $X$, we have

$$
\begin{aligned}
\left(\nabla_{X} Q\right) Y & =\nabla_{X} Q Y-Q\left(\nabla_{X} Y\right) \\
& =(1-\mu)\left[\left(\nabla_{X} \eta\right)(Y) \xi+\eta(Y) \nabla_{X} \xi\right] .
\end{aligned}
$$

Using (2.8) and (2.9),we get

$$
\left(\nabla_{X} Q\right) Y=(\mu-1)[g(X, Y) \xi+\eta(Y) X+2 \eta(X) \eta(Y) \xi] .
$$

Applying $\varphi^{2}$ on both sides of the above equation, we find

$$
\varphi^{2}\left(\nabla_{X} Q\right) Y=(\mu-1) \eta(Y) \varphi^{2} X .
$$

From (8.1) and (8.6), we obtain

$$
(\mu-1) \eta(Y) \varphi^{2} X=0,
$$

by virtue of (2.1) and (8.7), we find

$$
(\mu-1)[X+\eta(X) \xi]=0 .
$$

Taking inner product of (8.8) with respect to $U$, we get

$$
(\mu-1)[g(X, U)+\eta(X) \eta(U)]=0,
$$

from which it follows that $\mu=1$. From the relation (3.6), we obtain $\lambda=(2-n)$.

Thus, we can state the following theorem:

Theorem 8.1. On $\varphi$-Ricci symmetric Lorentzian para-Kenmotsu manifold admits a proper $\eta$-Ricci soliton with $\mu=1$ and $\lambda=(2-n)$.

From the above theorem we get:

Corollary 8.1. On a $\varphi$-Ricci symmetric Lorentzian para-Kenmotsu manifold, there is no Ricci solitons with potential vector field $\xi$. 


\section{9. $\quad \eta$-Ricci solitons on quasi-conformally Ricci semi-symmetric Lorentzian para-Kenmotsu manifolds}

In this section, we study $\eta$-Ricci solitons on conformally Ricci semi-symmetric Lorentzian para-Kenmotsu manifolds, that is,

$$
W \cdot S=0
$$

which implies

$$
(W(X, Y) \cdot S)(Z, U)=0
$$

From (9.2), we get

$$
S(W(X, Y) Z, U)+S(Z, W(X, Y) U)=0 .
$$

Using (3.3) in (9.3), we have

$$
\begin{aligned}
& (1-\lambda) g(W(X, Y) Z, U)+(1-\mu) \eta(W(X, Y) Z) \eta(U) \\
& +(1-\lambda) g(Z, W(X, Y) U)+(1-\mu)(W(X, Y) U) \eta(Z) \\
= & 0 .
\end{aligned}
$$

Taking $X=U=\xi$ in (9.4), we get

$$
\begin{aligned}
& (1-\lambda) g(W(\xi, Y) Z, \xi)-(1-\mu) \eta(W(\xi, Y) Z)+ \\
& (1-\lambda) g(Z, W(\xi, Y) \xi)+(1-\mu)(W(\xi, Y) \xi) \eta(Z) \\
= & 0 .
\end{aligned}
$$

From (2.18), we obtain

$$
W(\xi, Y) \xi)=\left[a+n b-b \lambda-\frac{r}{n}\left(\frac{a}{n-1}+2 b\right)\right][Y+\eta(Y) \xi] .
$$

By virtue of (9.6), we get

$$
\begin{aligned}
\eta(W(\xi, Y) Z) & =g(W(\xi, Y) Z, \xi) \\
& =-g(W(\xi, Y) \xi, Z) \\
& =-\left[a+n b-b \lambda-\frac{r}{n}\left(\frac{a}{n-1}+2 b\right)\right][g(Y, Z)+\eta(Y) \eta(Z)] .
\end{aligned}
$$

Also, from (9.7), we find

$$
\eta(W(\xi, Y) \xi)=0 .
$$

Making use of (9.6), (9.7) and (9.8) in (9.5), we have

$$
(1-\mu)\left[a+n b-b \lambda-\frac{r}{n}\left(\frac{a}{n-1}+2 b\right)\right][g(Y, Z)+\eta(Y) \eta(Z)]=0,
$$


either

$$
1-\mu=0, \text { or }\left[a+n b-b \lambda-\frac{r}{n}\left(\frac{a}{n-1}+2 b\right)\right]=0,
$$

either $\mu=1$ or $\left[a+n b-b \lambda-\frac{r}{n}\left(\frac{a}{n-1}+2 b\right)\right]=0$,

Case I : if $\mu=1$ then from (3.6), we find $\lambda=2-n$.

Case II : if $\left[a+n b-b \lambda-\frac{r}{n}\left(\frac{a}{n-1}+2 b\right)\right]=0$,

implies that $\lambda=\frac{a}{b}\left(1-\frac{r}{n(n-1)}\right)+\left(n-\frac{2 r}{n}\right)$. From the relation (3.6), we obtain $\mu=\frac{a}{b}\left(1-\frac{r}{n(n-1)}\right)+\left(2 n-\frac{2 r}{n}-1\right)$.

Thus, we can state the following theorem:

Theorem 9.1. If a conformally Ricci semi-symmetric Lorentzian para-Kenmotsu manifold admits a proper $\eta$-Ricci soliton, then $\mu=1$ and $\lambda=2-n$ or $\lambda=$ $\frac{a}{b}\left(1-\frac{r}{n(n-1)}\right)+\left(n-\frac{2 r}{n}\right)$ and $\mu=\frac{a}{b}\left(1-\frac{r}{n(n-1)}\right)+\left(2 n-\frac{2 r}{n}-1\right)$.

From the above theorem we have the following:

Corollary 9.1. On conformally Ricci semi-symmetric Lorentzian para-Kenmotsu manifold, there is no Ricci solitons with potential vector field $\xi$.

\section{Example}

Now, we consider the 3-dimensional manifold

$$
M=\left\{(x, y, z) \in R^{3}: z \neq 0,\right\}
$$

where $x, y, z$ are the standard coordinates in $R^{3}$.

The vector fields

$$
e_{1}=z \frac{\partial}{\partial x}, e_{2}=z \frac{\partial}{\partial y}, \text { and } e_{3}=z \frac{\partial}{\partial z}=\xi
$$

are linearly independent at each point of $M$ and $\alpha$ is constant.

Let $g$ be the Lorentzian metric defined by

$$
\begin{aligned}
& g\left(e_{1}, e_{3}\right)=g\left(e_{2}, e_{3}\right)=g\left(e_{1}, e_{2}\right)=0, \\
& g\left(e_{1}, e_{1}\right)=g\left(e_{2}, e_{2}\right)=1, g\left(e_{3}, e_{3}\right)=-1 .
\end{aligned}
$$

Let $\eta$ be the 1-form defined by

$$
\eta(Z)=g\left(Z, e_{3}\right)=g(Z, \xi),
$$

for any vector field $Z$ on $M$. 
Let $\varphi$ be the $(1,1)$-tensor field defined by

$$
\varphi\left(e_{1}\right)=-e_{2}, \varphi\left(e_{2}\right)=-e_{1}, \varphi\left(e_{3}\right)=0 .
$$

Then, using the linearity of $\varphi$ and $g$, we have

$$
\begin{aligned}
\eta\left(e_{3}\right) & =-1, \varphi^{2} Z=Z+\eta(Z) e_{3}, \\
g(\varphi Z, \varphi W) & =g(Z, W)+\eta(Z) \eta(W),
\end{aligned}
$$

for any vector field $Z, W$ on $M$.

It is easy to see that

$$
\eta\left(e_{1}\right)=0, \eta\left(e_{2}\right)=0, \eta\left(e_{3}\right)=-1 .
$$

Thus for $e_{3}=\xi$, the structure $(\varphi, \xi, \eta, g)$ defines a Lorentzian almost paracontact metric structure on $M$. [1]

Let $\nabla$ be the Levi-Civita connection with respect to the Lorentzian metric $g$. Then we have

$$
\text { (10.6) } \quad\left[e_{1}, e_{2}\right]=0,\left[e_{1}, e_{3}\right]=-e_{1},\left[e_{2}, e_{3}\right]=-e_{2} \text {. }
$$

Using Koszul's formula for Levi-Civita connection $\nabla$ with respect to $g$, i.e.,

$$
\begin{aligned}
2 g\left(\nabla_{X} Y, Z\right)= & X g(Y, Z)+Y g(Z, X)-Z g(X, Y) \\
& -g(X,[Y, Z])-g(Y,[X, Z]) \\
& +g(Z,[X, Y]),
\end{aligned}
$$

One can easily calculate

$$
\begin{gathered}
\nabla_{e_{1}} e_{1}=-e_{3}, \quad \nabla_{e_{1}} e_{2}=0, \quad \nabla_{e_{1}} e_{3}=-e_{1}, \\
\nabla_{e_{2}} e_{1}=0, \quad \nabla_{e_{2}} e_{2}=-e_{3}, \quad \nabla_{e_{2}} e_{3}=-e_{2}, \\
\nabla_{e_{3}} e_{1}=0, \quad \nabla_{e_{3}} e_{2}=0, \quad \nabla_{e_{3}} e_{3}=0 .
\end{gathered}
$$

From the above calculations, we see that the manifold under consideration satisfies $\nabla$, i.e.,

$$
\nabla_{Z} \xi=-Z-\eta(Z) \xi \text {, and }\left(\nabla_{Z} \varphi\right) W=-g(\varphi Z, W) \xi-\eta(W) \varphi Z .
$$

Also, the Riemannian curvature tensor $R$ is given by

$$
R(X, Y) Z=\nabla_{X} \nabla_{Y} Z-\nabla_{Y} \nabla_{X} Z-\nabla_{[X, Y]} Z .
$$

Then

$$
\begin{gathered}
R\left(e_{1}, e_{2}\right) e_{2}=e_{1}, R\left(e_{1}, e_{3}\right) e_{3}=-e_{1}, R\left(e_{1}, e_{2}\right) e_{1}=-e_{2}, \\
R\left(e_{2}, e_{3}\right) e_{1}=0, R\left(e_{1}, e_{3}\right) e_{2}=0, R\left(e_{1}, e_{2}\right) e_{3}=0, \\
R\left(e_{2}, e_{3}\right) e_{3}=-e_{2}, R\left(e_{1}, e_{3}\right) e_{1}=-e_{3}, R\left(e_{2}, e_{3}\right) e_{2}=-e_{3} .
\end{gathered}
$$

Then, the Ricci tensor $S$ is given by

$$
S\left(e_{1}, e_{1}\right)=S\left(e_{2}, e_{2}\right)=2, \quad S\left(e_{3}, e_{3}\right)=-2 .
$$

From (3.5), we obtain $S\left(e_{1}, e_{1}\right)=S\left(e_{2}, e_{2}\right)=1-\lambda$ and $S\left(e_{3}, e_{3}\right)=\lambda-\mu$, therefore $\lambda=-1$ and $\mu=1$. The data $(g, \xi, \lambda, \mu)$ for $\lambda=-1$

and $\mu=1$ defines an $\eta$-Ricci soliton on the Lorentzian para Kenmotsu manifold $M$. 


\section{REF E R E N C E S}

1. A. Haseeb and R. Prasad, Certain Results on Lorentzian Para-Kenmotsu Manifolds, Bol. Soc. Paran. Mat., (2021) no. 3, 201-220.

2. A. GRAY, Einstein-like manifolds which are not Einstein, Geom. Dedicata 7 (1978), no. 3, 259-280.

3. A. M. Blaga, $\eta$-Ricci solitons on Lorentzian para-Sasakian manifolds, Filomat, 30 (2016), no. 2, 489-496.

4. A. Singh and S. KISHOR, Some types of $\eta$-Ricci solitons on Lorentzian para-Sasakian manifolds, Facta Univ. (NIS) 33(2), 217-230 (2018).

5. C. CÁlin and M. Crasmareanu, $\eta$-Ricci solitons on Hopf hypersurfaces in complex space forms, Rev. Roumaine Math. Pures Appl., 57 (2012), no. 1, 55-63.

6. C. Cálin and M. Crasmareanu, From the Eisenhart problem to Ricci solitons in f-Kenmotsu manifolds, Bull. Malaysian Math. Sci. Soc., 33 (2010), no. 3, 361-368.

7. D. FriedAn, Non linear models in $2+\epsilon$ dimensions, Ann. Phys., 163 (1985), 318-419.

8. D. G. Prakasha and B. S. Hadimani, $\eta$-Ricci solitons on para-Sasakian manifolds, J. Geom., 108 (2017), 383-392.

9. J. T. Сho and M. KimurA, Ricci solitons and real hypersurfaces in a complex space form, Tohoku Math. J., 61 (2009), no. 2, 205-212.

10. K. OndA, Lorentz Ricci solitons on 3-dimensional Lie groups, Geom. Dedicata 147 (2010), 313-322.

11. K. YAno and M. Kon, Structures on Manifolds, Ser. Pure Math. 3, World Scientific, Singapore, 1984.

12. K. YANO and S. SAWAKI, Riemannian manifolds admitting a conformal transformation group, J. Differential Geom. 2 (1968), 161-184.

13. M. Ali and Z. Ahsan, Quasi-conformal curvature tensor for the spacetime of general relativity, Palest. J. Math. 4 (2015), no. 1, 234-241.

14. M. Brozos-VÀzquez, G. Calvaruso, E. Garcìa-Rìo and S. Gavino-Fernàndez, Three dimensional Lorentzian homogeneous Ricci solitons, Israel J. Math. 188 (2012), 385-403.

15. P. Alegre, Slant submanifolds of Lorentzian Sasakian and Para Sasakian manifolds, Taiwanese J. Math. 17, 897-910, (2013).

16. P. Majhi, U. C. De and D. KAR, $\eta$-Ricci solitons on Sasakian 3-Manifolds, An. Univ. Vest. Timis. Ser. Mat.-Inform. LV, 2 (2017), 143-156.

17. R. S. Hamilton, Three-manifolds with positive Ricci curvature, J. Diff . Geom., 17 (1982), no. 2, 255-306.

18. R. S. Hamilton, The Ricci flow on surfaces, Mathematics and general relativity, (Contemp. Math. Santa Cruz, CA, 1986), American Math. Soc., (1988), 237-263.

19. S. Deshmukh, H. Alodan and H. Al-Sodais, A Note on Ricci Soliton, Balkan J. Geom. Appl., 16 (2011), no. 1, 48-55.

20. S. Kishor and A. Singh, Curvature Properties of $\eta$-Ricci Solitons on Para-Kenmotsu Manifolds, Kyungpook Mathematical Journal 59(1):149-161 (2019).

21. T. IVEY, Ricci solitons on compact 3-manifolds, Diff. Geom. Appl., 3 (1993), 301-307. 
22. T. Chave and G. Valent, Quasi-Einstein metrics and their renormalizability properties, Helv. Phys. Acta., 69 (1996), 344-347.

23. T. Chave and G. VALEnt, On a class of compact and non-compact quasi-Einstein metrics and their renormalizability properties, Nuclear Phys. B., 478 (1996), 758-778.

24. U. C. DE, Ricci solitons and gradient Ricci solitons in a P-Sasakian manifold, The Aligarh Bull. of Maths., 29 (2010), no.1, 29-33.

25. U. C. De and A. K. Mondal, 3-dimensional quasi-Sasakian manifolds and Ricci solitons, SUT J. Math., 48 (2012), no. 1, 71-81.

26. U. C. De, M. Turan, A. YILDIZ and A. De, Ricci solitons and gradient Ricci solitons on 3-dimensional normal almost contact metric manifolds, Publ. Math. Debrecen, 80 (2012), no. 1-2, 127-142.

27. U. C. De and A. Sarkar, On $\phi$-Ricci symmetric Sasakian manifolds, Proc. Jangjeon Math. Soc.11 (2008), 47-52.

28. W. Batat, M. Brozos-VÀzquez, E. Garcìa-Rìo ; and S. Gavino-Fernàndez, Ricci solitons on Lorentzian manifolds with large isometry groups, Bull. London Math. Soc. 43 (2011), no. 6, 1219-1227. 